\title{
Quantum Transport Thermometry for Electrons in Graphene
}

\author{
K. Kechedzhi, ${ }^{1}$ D. W. Horsell, ${ }^{2}$ F. V. Tikhonenko, ${ }^{2}$ A. K. Savchenko, ${ }^{2}$ R. V. Gorbachev, ${ }^{2}$ I. V. Lerner, ${ }^{3}$ and V. I. Fal'ko ${ }^{1}$ \\ ${ }^{1}$ Department of Physics, Lancaster University, Lancaster, LA1 4YB, United Kingdom \\ ${ }^{2}$ School of Physics, University of Exeter, Stocker Road, Exeter, EX4 4QL, United Kingdom \\ ${ }^{3}$ School of Physics and Astronomy, University of Birmingham, Egbaston, Birmingham B15 2TT, United Kingdom
}

(Received 26 August 2008; published 9 February 2009)

\begin{abstract}
We propose a method of measuring the electron temperature $T_{e}$ in mesoscopic conductors and demonstrate experimentally its applicability to micron-size graphene devices in the linear-response regime ( $T_{e} \approx T$, the bath temperature). The method can be especially useful in case of overheating, $T_{e}>$ $T$. It is based on analysis of the correlation function of mesoscopic conductance fluctuations. Although the fluctuation amplitude strongly depends on the details of electron scattering in graphene, we show that $T_{e}$ extracted from the correlation function is insensitive to these details.
\end{abstract}

DOI: 10.1103/PhysRevLett.102.066801

Graphene is an atomically thin graphite layer [1,2] recently used in field-effect transistors [3]. In graphenebased semiconductor devices phonons are poorly coupled to the environment since the mass of carbon atoms is typically smaller than that of atoms in the underlying substrate, making the overheating of graphene structures a likely event at high currents. This raises a question of how to measure the temperature of electrons in graphene. Since classical conductivity in graphene has a very weak temperature dependence at low and intermediate temperatures [4], extracting the electron temperature from transport measurements requires analyzing more subtle quantum effects. One possibility would be to analyze the decoherence rate $\tau_{\varphi}^{-1}$ using the weak-localization (WL) effects in magneto-resistance [5,6]. However, it was shown theoretically [7] and confirmed experimentally [8] that the WL in graphene reveals itself in a rather complicated way due to the influence of intervalley scattering and the disorder which breaks the sublattice symmetry. Thus WL does not offer an easy way of measuring the electron temperature $T_{e}$. Another possibility would be to exploit the temperature dependence of the amplitude of universal conductance fluctuations (UCF) $[5,9,10]$. Unfortunately, a quantitative implementation of such analysis is hindered by the necessity to both account for the temperature dependence of $\tau_{\varphi}^{-1}$ and attribute a definite symmetry class to a particular graphene-based device [8,11-13]. However, it has been noticed that the correlation functions of random UCF dependence on magnetic field $B$ and the Fermi energy $\varepsilon_{F}$ provide useful information about subtle spectral characteristics of a disordered conductor [14].

In this Letter we propose a method of correlationfunction thermometry of mesoscopic conductors and demonstrate experimentally its applicability to graphene-based micron-size devices. Although the UCF in graphene differ in detail [11-13] from those in other mesoscopic conductors, the proposed method is robust and independent of such details. It is based on the analysis of the normalized
PACS numbers: 73.23.-b, 72.15.Rn, 73.43.Qt, 81.05.Uw

correlation function $F_{n}(\Delta)$ of conductance fluctuations, $\delta G=G-\langle G\rangle$, as a function of the Fermi energy (using a wide range of the UCF magnetofingerprints for statistically representative averaging [15]):

$$
F_{n}(\Delta) \equiv \frac{F(\Delta)}{F(0)}, \quad F(\Delta) \equiv\left\langle\left\langle\delta G\left(\varepsilon_{F}\right) \delta G\left(\varepsilon_{F}+\Delta\right)\right\rangle\right\rangle .
$$

This function depends on the electron temperature $T_{e}$ which governs the thermal broadening of the Fermi distribution. We show that for a quasi-1D wire the width $\Delta_{c}$ of $F_{n}$ (taken at the half-maximum) is defined by $T_{e}$ :

$$
\Delta_{c} \approx 2.7 k_{B} T_{e} .
$$

This expression allows one to determine $T_{e}$ by extracting $\Delta_{c}$ from measuring the correlation function (1). The result is valid with accuracy of about $10 \%$ provided that the following conditions are fulfilled:

$$
\begin{aligned}
& L_{T} \ll \min \left(L_{\varphi}, L_{x}\right), \\
& L_{y} \ll \min \left(L_{\varphi}, L_{x}\right),
\end{aligned}
$$

where $L_{T} \equiv \sqrt{\hbar D / k_{B} T_{e}}$ is the thermal smearing length and $L_{\varphi} \equiv \sqrt{D \tau_{\varphi}}$ is the dephasing length, $D$ is the diffusion coefficient. Inequality (3a) defines an experimentally relevant "high- $T$ " regime (which may extend well below $1 \mathrm{~K}$ ) and is paramount for the method to work. Inequality (3b) is less demanding: one can use Eq. (2) to determine $T_{e}$ also for a 2D sample with aspect ratio $a \equiv L_{x} / L_{y} \sim 1$ up to $L_{\varphi} / L_{y} \sim 1$, albeit with lesser accuracy [17].

We have experimentally tested the method in four graphene flakes in the regime (3), using low-current measurements to avoid electron overheating. Then $T_{e}$ should coincide with the bath temperature $T$. We show the results in Fig. 1, where $T_{e}$ xtracted from Eq. (2) and the directly measured $T$ are, indeed, in good agreement. 


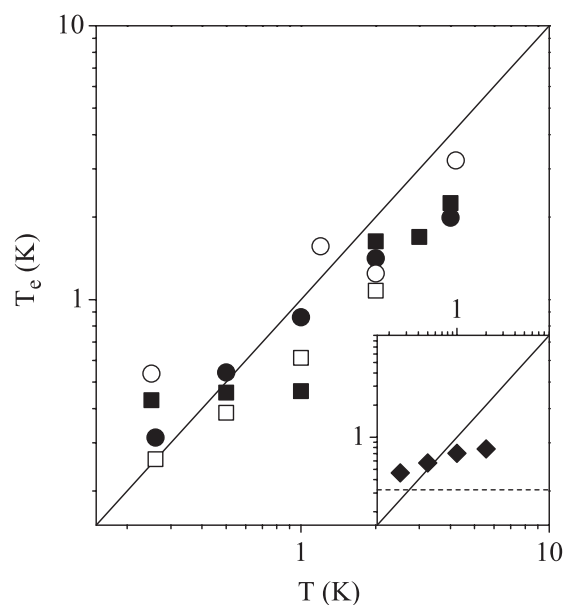

FIG. 1. The electronic temperature $T_{e}$, determined via Eq. (2), as a function of the bath temperature, $T$, for four graphene flakes (F1: open circles, F2: open squares, B1: closed circles, B2: closed squares; see Table I). The inset shows $\Delta_{c} / 2.7$ for sample D with $L_{T} \sim L_{x}$, i.e., not in the regime (3); the dashed horizontal line shows the Thouless energy, $h / \tau_{D}$.

Below we first derive our main result, Eq. (2), then proceed with its numerical testing and finally discuss experimental results in more detail.

The brackets $\langle\langle\ldots\rangle\rangle$ in Eq. (1) stand for both the ensemble and thermal averaging. This equation can be explicitly represented $[9,10]$ as the following convolution:

$$
\begin{aligned}
F(\Delta) & =\int d \varepsilon K(\varepsilon, \Delta) F(\varepsilon), \\
K(\varepsilon, \Delta) & =\left(\frac{4 e^{2}}{h}\right)^{2} \int d E f^{\prime}\left(E, \varepsilon_{F}\right) f^{\prime}\left(E+\varepsilon, \varepsilon_{F}+\Delta\right) .
\end{aligned}
$$

Here $\mathcal{F}(\varepsilon) \equiv\langle\delta G(E) \delta G(E+\varepsilon)\rangle$ is the ensembleaveraged correlator of conductance fluctuations at different energies and $K$ is the thermal broadening factor, where the energy derivative of the Fermi distribution function is $f^{\prime}\left(E, \varepsilon_{F}\right)=-1 /\left(4 k_{B} T_{e}\right) \cosh ^{-2}\left[\left(E-\varepsilon_{F}\right) /\left(2 k_{B} T_{e}\right)\right]$. The standard diagrams for $\mathcal{F}(\varepsilon)$ in the lowest order in $\hbar /\left(p_{F} \ell\right) \ll 1(\ell$ is the electron mean-free path) are shown in Fig. 2. Structurally, they coincide with the diagrams describing mesoscopic fluctuations in usual conductors $[9,18]$ but the so-called Hikami boxes are different [1113] because of graphene-specific features: the linear dis-
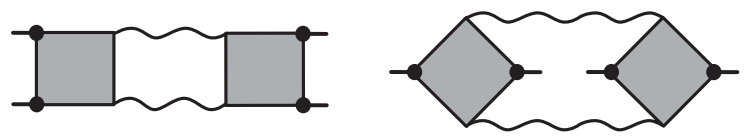

FIG. 2. The diagrams which contribute to the main order in the diagrammatic expansion of the conductivity-conductivity correlation function. The wavy lines stand for the propagators of the diffusion modes describing motion at length scales $\gg \ell$; shaded blocks stand for Hikami boxes, describing motion at length scales $\sim \ell$. persion law, chirality of the carriers and valley degeneracy. These features, being paramount for a quantitative description of the UCF in graphene [11-13], have no impact on calculating $F_{n}$. We will show this by analyzing first a narrow graphene wire, Eq. (3b), with a strong intervalley scattering (induced, e.g., by atomically sharp disorder). Then the part of the correlator $\mathcal{F}$ which contributes to $F_{n}$ can be written via the "valley-singlet" diffusion propagators $\mathcal{D}_{n m}$, neglecting the "valley-triplet" modes (see Refs. $[7,11]$ for the appropriate classification of the diffusion modes in graphene):

$$
\begin{aligned}
\mathcal{F}(\varepsilon) & =\sum_{n, m}\left(\left|\mathcal{D}_{n m}\right|^{2}+\frac{1}{2} \Re\left[\mathcal{D}_{n m}\right]^{2}\right), \\
\mathcal{D}_{n m} & \equiv\left[-\frac{i}{\hbar} \varepsilon \tau_{D}+(\pi n)^{2}+(a \pi m)^{2}+\frac{\tau_{D}}{\tau_{\varphi}}\right]^{-1},
\end{aligned}
$$

where $a=L_{x} / L_{y}$ and $\tau_{D}=L_{x}^{2} / D$. Under the conditions (3) the sum in Eq. (5a) is dominated by the $m=0$ term. There we have taken into account only the diffusion modes and neglected the Cooperons. This corresponds to the regime of suppressed WL, when the magnetic flux through the area of order $L_{\varphi}^{2}$ is much bigger than the flux quantum. In this regime a wider range of statistical data is available due to the averaging over magnetic fields [15].

In order to get an asymptotic analytical expression for the correlation function $F_{n}(\Delta)$ we assume that in addition to Eq. (3) the dephasing is sufficiently strong, i.e., $L_{x} \gg$ $L_{\varphi}$. Keeping only the term with $m=0$ and performing the summation over $n$, we arrive at

$$
\mathcal{F}(\varepsilon)=\frac{1}{2 \sqrt{2}}\left(\frac{L_{\varphi}}{L_{x}}\right)^{3} \frac{3 t^{2}+t+2}{t^{3} \sqrt{t+1}}-\left(\frac{L_{\varphi}}{L_{x}}\right)^{4} \frac{t^{2}+2}{t^{4}},
$$

where $t \equiv \sqrt{\left(\varepsilon \tau_{\varphi} / \hbar\right)^{2}+1}$. The correlator $\mathcal{F}(\varepsilon)$ in Eq. (6) is a sharply peaked function of $\varepsilon$ with maximum at $\varepsilon=0$ and width $\hbar / \tau_{\varphi}$. In contrast, the thermal broadening factor $K(\varepsilon, \Delta)$ in Eq. (4) has a broad peak around $\varepsilon=\Delta$ with the width of the order of $k_{B} T_{e} \gg \hbar / \tau_{\varphi}$, according to Eq. (3a). Therefore the integration over $\varepsilon$ in Eq. (4) can be performed using the mean value theorem, i.e., taking $K(0, \Delta)$ out of the integral. As a result the normalized correlation function becomes independent of the microscopic details contained in $\mathcal{F}(\varepsilon)$ :

$$
F_{n}(\Delta)=\frac{K(0, \Delta)}{K(0,0)}=\frac{3(\theta \operatorname{coth} \theta-1)}{\sinh ^{2} \theta}, \quad \theta \equiv \frac{\Delta}{2 k_{B} T_{e}} .
$$

The width of this function at the half-maximum is $\theta_{c}=$ 1.36 which results in Eq. (2). We stress again that this result is truly universal: a precise form of $\mathcal{F}(\varepsilon)$ is irrelevant for $F_{n}(\Delta)$ in Eq. (7). The only requirement for its validity is that the function $\mathcal{F}(\varepsilon)$ in Eq. (4) is sharply peaked compared to $K(\varepsilon, \Delta)$. This remains valid under the condition (3a) for any dephasing, $L_{\varphi} \lesssim L_{x}$, and allowing for all the 
diffusion modes in graphene or, indeed, in any other mesoscopic disordered conductor.

We have checked this numerically, calculating $F_{n}(\Delta)$ for a wide range of $T$ and $\tau_{\varphi}, 0.01 \leq L_{T} / L_{\varphi} \leq 0.3$ with $L_{\varphi} \leq$ $L_{x}$, and at various values of the symmetry breaking parameters. We have also considered a case of smooth disorder, taking into account the valley-triplet diffusion channels [neglected in the analytical calculations in Eqs. (5) and (6)]. A few representative examples are plotted in Fig. 3. The values of $\Delta_{c}$ lie within a narrow interval, $2.7 \leq \Delta_{c} / k_{B} T_{e} \leq 2.9$, close to the asymptotic value 2.7 of Eq. (2). This shows that the proposed method works in the quasi-1D case with accuracy of about $10 \%$. We have also performed a similar analysis of $2 \mathrm{D}$ samples, calculating $F_{n}(\Delta)$ for the same range of parameters as for wires. The results plotted in the inset to Fig. 3 indicate that $\Delta_{c} \approx$ $3 k_{B} T_{e}$; i.e., the method can still be applied albeit with accuracy of about $25 \%$.

We have tested the feasibility of the proposed method in graphene-based structures. To this end, we have experimentally determined the width of the correlation function (1) and thus the electron temperature $T_{e}$, Eq. (2). We have compared $T_{e}$ to the bath temperature $T$ in low-current measurements, i.e., in the regime when graphene is not overheated, and found them to be in satisfactory agreement, Fig. 1. The experimental samples used for correlation-function thermometry are monolayer graphene flakes created by mechanical exfoliation [20] on a $n^{+} \mathrm{Si}$ substrate covered by $300 \mathrm{~nm}$ of $\mathrm{SiO}_{2}$. The flakes are connected electrically by two $\mathrm{Au} / \mathrm{Cr}$ contacts in the circuit shown in the inset to Fig. 4 and thermally anchored within the ${ }^{3} \mathrm{He}$ pot of a sorption-pumped cryostat. The ac current driven through the sample was $1 \mathrm{nA}$ (at $\sim 10 \mathrm{~Hz}$ ). Heating

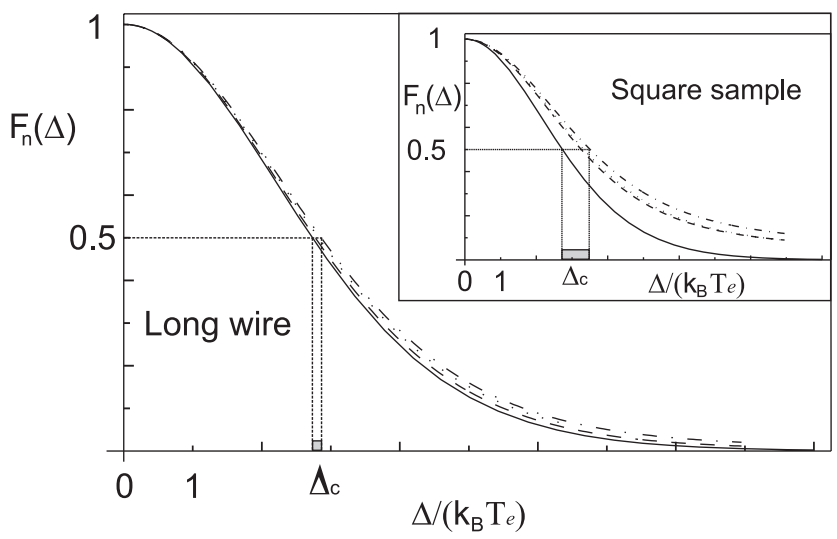

FIG. 3. The normalized correlation function, $F_{n}(\Delta)$, for a wire under conditions (3). The solid line corresponds to the asymptotic Eq. (7). The dotted, dashed, and dash-dotted lines correspond to the numerical integration of Eqs. (4) with $\alpha=0.01$, $\beta=0.3 ; \alpha=\beta=0.1 ; \alpha=0.1, \beta=0.3$, respectively $(\alpha \equiv$ $\left.L_{T} / L_{x}, \beta \equiv L_{T} / L_{\varphi}\right)$. The width at half maximum is $\Delta_{c} \approx$ $(2.8 \pm 0.1) k_{B} T_{e}$. The inset shows $F_{n}(\Delta)$ for a square sample where each line has the same values of $\alpha$ and $\beta$. by the current was detected by measuring the mesoscopic fluctuations, and this current was reduced until it had no effect on the fluctuations. The Fermi energy was controlled by a gate voltage $V_{g}$ applied between the substrate and the flake [21]. Samples characteristics are described in Table I. The dephasing rate in all samples was determined in a way similar to that in [8] from a fit of the magnetic field dependence of the sample conductivity to the theory of weak localization in graphene [7]. For these samples at high carrier density, $\sim 10^{12} \mathrm{~cm}^{-2}, \min \left(L_{x}, L_{\varphi}\right)$ satisfies the conditions (3) for the applicability of the method.

Mesoscopic fluctuations of the conductance $G$ occur in all samples as a function of both magnetic field and Fermi energy, Fig. 4(a), and are reproducible for the forward and backward sweeps of $\varepsilon_{F}$ and $B$. The amplitude and correlation function both depend on the bath temperature $T$ over the full temperature range of the experiment $(0.25-20 \mathrm{~K})$. A range of Fermi energies is chosen such that the average resistance does not change significantly over this range and contains sufficient number $(>100)$ of fluctuations for averaging (at $T \geqslant 10 \mathrm{~K}$ such a requirement cannot be satisfied so we restrict analysis to $T<10 \mathrm{~K}$ ). We determine $F_{n}(\Delta)$, Fig. 4(b), and its width at half maximum $\Delta_{c}$ performing measurements over the magnetic field range $50 \mathrm{mT} \lesssim$ $B \lesssim 300 \mathrm{mT}$ [15]. It is shown in Fig. 1 as a function of the bath temperature and agrees with the theoretical relation, Eq. (2), with $T_{e} \approx T$. For comparison, the inset to

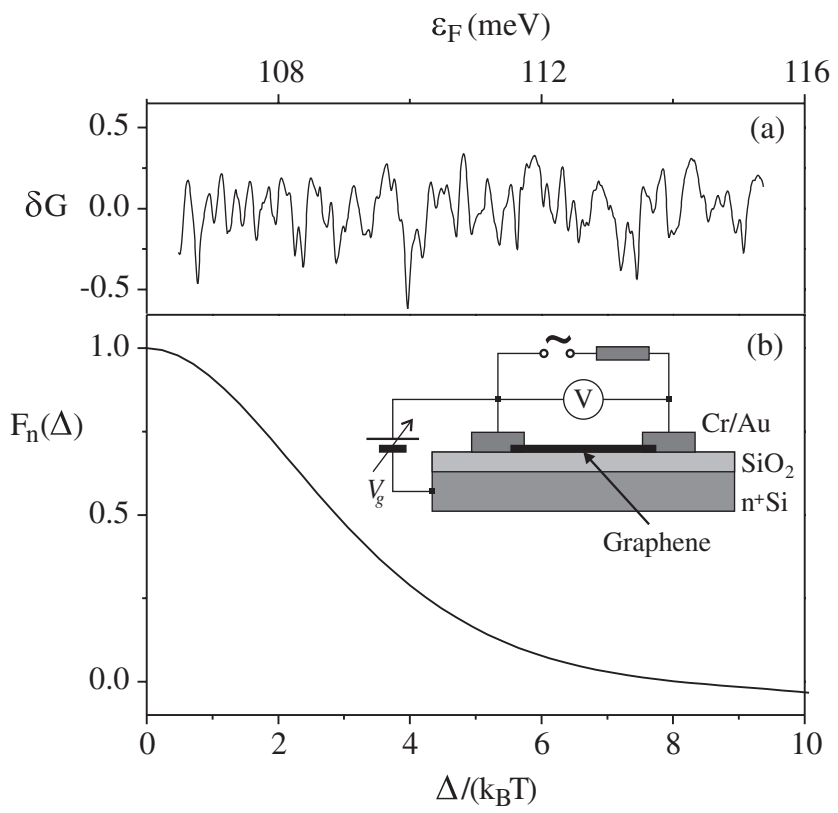

FIG. 4. (a) Typical fingerprint of $\delta G$ (normalized by $e^{2} / h$ ) for sample $\mathrm{B} 1$ at $T=0.25 \mathrm{~K}$ and $B=90 \mathrm{mT}$ as a function of Fermi energy (controlled by the gate voltage [21].) (b) Correlation function for sample B1 at $T=0.25 \mathrm{~K}$. The inset sketches the circuit used in the experiment. The resistor has resistance much greater than that of the graphene flake to maintain a fixed ac current of $1 \mathrm{nA}$. 
TABLE I. Characteristics of the samples used for the correlation-function thermometry, Fig. 1. The values of $L_{\varphi}$ are given at $T=0.26 \mathrm{~K}$; for the last three samples, where $L_{\varphi}>L_{x}$, it cannot be determined from the WL measurements.

\begin{tabular}{lccccc}
\hline \hline & $L_{x}(\mu \mathrm{m})$ & $L_{y}(\mu \mathrm{m})$ & $L_{\varphi}(\mu \mathrm{m})$ & $\ell(\mathrm{nm})$ & $n\left(10^{12} \mathrm{~cm}^{-2}\right)$ \\
\hline D & 1.4 & 1.4 & 1.2 & 70 & 1.4 \\
$\mathrm{~F} 1$ & 4.1 & 1.8 & 1.7 & 70 & 1.4 \\
$\mathrm{~F} 2$ & 3.8 & 1.8 & $>L_{x}$ & 120 & 0.7 \\
$\mathrm{~B} 1$ & 3.7 & 0.3 & $>L_{x}$ & 80 & 0.9 \\
$\mathrm{~B} 2$ & 2.0 & 0.3 & $>L_{x}$ & 50 & 0.9 \\
\hline \hline
\end{tabular}

Fig. 1 shows that $\Delta_{c}$ for sample D (which is not in the "high-temperature" regime of Eq. (3a)) saturates at $h / \tau_{D}$.

In conclusion, we have proposed to use the correlation function of mesoscopic fluctuations in disordered samples for determining the electron temperature from its width. We have shown that the method is universal and independent of microscopic details of a disordered mesoscopic sample: Eq. (2) holds for any sample in regime (3a), determining $T_{e}$ with good accuracy in quasi-1D samples, (3b). We have confirmed the viability of the method performing measurements on graphene devices in the lowcurrent regime, when $T_{e} \approx T$. For future applications, the method may be especially useful for graphene devices at higher currents, where overheating is likely to arise from inefficient thermal contact with the environment.

We acknowledge support from the EPSRC grant EP/ D031109, the Lancaster-EPSRC Portfolio Partnership EP/C511743, and ESF FoNE CRP "SpiCo."

[1] P. R. Wallace, Phys. Rev. 71, 622 (1947).

[2] J. W. McClure, Phys. Rev. 104, 666 (1956).

[3] A. K. Geim and K. S. Novoselov, Nature Mater. 6, 183 (2007).

[4] S. V. Morozov, K. S. Novoselov, M. I. Katsnelson, F. Schedin, D.C. Elias, J.A. Jaszczak, and A. K. Geim, Phys. Rev. Lett. 100, 016602 (2008).

[5] I. L. Aleiner, B.L. Altshuler, and M.E. Gershenson, Waves Random Media 9, 201 (1999); I. L. Aleiner and Ya. M. Blanter, Phys. Rev. B 65, 115317 (2002).

[6] C. M. Marcus, R. M. Westervelt, P. F. Hopkins, and A. C. Gossard, Phys. Rev. B 48, 2460 (1993).

[7] E. McCann, K. Kechedzhi, V. I. Fal'ko, H. Suzuura, T. Ando, and B.L. Altshuler, Phys. Rev. Lett. 97, 146805 (2006); K. Kechedzhi, V. I. Fal'ko, E. McCann, and B. L. Altshuler, Phys. Rev. Lett. 98, 176806 (2007).
[8] F. V. Tikhonenko, D. W. Horsell, R. V. Gorbachev, and A. K. Savchenko, Phys. Rev. Lett. 100, 056802 (2008).

[9] B. L. Altshuler and D. E. Khmelnitskii, JETP Lett. 42, 476 (1985).

[10] P. A. Lee, A. D. Stone, and H. Fukuyama, Phys. Rev. B 35, 1039 (1987).

[11] K. Kechedzhi, O. Kashuba, and V. I. Fal'ko, Phys. Rev. B 77, 193403 (2008).

[12] M. Y. Kharitonov and K. B. Efetov, Phys. Rev. B 78, 033404 (2008).

[13] J. Wurm, A. Rycerz, I. Adagideli, M. Wimmer, K. Richter, and H. U. Baranger, arXiv:0808.1008 [Phys. Rev. Lett. (to be published)].

[14] T. Schmidt, P. König, E. McCann, V.I. Fal'ko, and R. J. Haug, Phys. Rev. Lett. 86, 276 (2001); J. Könemann, P. König, T. Schmidt, E. McCann, V.I. Fal'ko, and R. J. Haug, Phys. Rev. B 64, 155314 (2001).

[15] In measurements, $\left\langle\delta G \delta G^{\prime}\right\rangle$ is the correlation function of the fluctuations of conductance $\delta G\left(\varepsilon_{F}\right)$ averaged over a wide range of Fermi energies and magnetic fields. For given $\varepsilon_{F}$, the averaging over magnetic field is done by extracting from experimental data the autocorrelator $\left\langle\delta G \delta G^{\prime}\right\rangle=\int_{B}^{B+B_{0}} \frac{d B^{\prime}}{B_{0}} G\left(B^{\prime}, \varepsilon_{F}\right) G\left(B^{\prime}, \varepsilon_{F}+\Delta\right)$. In our theoretical analysis we use the standard substitution of such an average by the disorder ensemble averaging, based upon the ergodicity hypothesis [16].

[16] B. L. Altshuler, V. E. Kravtsov, and I. V. Lerner, JETP Lett. 43, 441 (1986); O. Tsyplyatyev, I. L. Aleiner, V. I. Fal'ko, and I. V. Lerner, Phys. Rev. B 68, 121301(R) (2003).

[17] In case of overheating the temperature distribution in the sample may be inhomogeneous; then Eq. (2) determines the average electron temperature in the sample.

[18] Note in passing that only diagrams with two diffusion modes contribute in the lowest order. The diagrams with three or four diffusion modes, taken into account in [10], mutually cancel as they originate from the disorder averaging of the $G^{R} G^{R}$ and $G^{A} G^{A}$ contribution to the conductance which vanish in each given realization of disorder [19] $\left(G^{R / A}\right.$ are the retarded or advanced Green's functions).

[19] H. U. Baranger and A. D. Stone, Phys. Rev. B 40, 8169 (1989).

[20] K. S. Novoselov, A. K. Geim, S. V. Morozov, D. Jiang, Y. Zhang, S. V. Dubonos, I. V. Grigorieva, and A. A. Firsov, Science 306, 666 (2004).

[21] In graphene with the linear dispersion law $\varepsilon=v p$, this relation is $\varepsilon_{F}=2 \hbar v \sqrt{\pi V_{g} C / e}$, where $C$ is the capacitance per unit area between the gate electrode and graphene. For our geometry, this relation reduces to $\varepsilon_{F}=$ $30 V_{g}^{1 / 2}$ where $V_{g}$ is measured in Volts and $\varepsilon_{F}$ in meV. 